https://helda.helsinki.fi

Circulating tumor DNA (ctDNA) in precision oncology of ovarian cancer

\title{
Oikkonen, Jaana
}

2019-12

Oikkonen , J \& Hautaniemi , S 2019 , ' Circulating tumor DNA (ctDNA) in precision oncology of ovarian cancer ' , Pharmacogenomics , vol. 20 , no. 18 , pp. 1251-1253 . https://doi.org/10.2217/pgs-2019-0126

http://hdl.handle.net/10138/324232

https://doi.org/10.2217/pgs-2019-0126

acceptedVersion

Downloaded from Helda, University of Helsinki institutional repository.

This is an electronic reprint of the original article.

This reprint may differ from the original in pagination and typographic detail.

Please cite the original version. 


\title{
Circulating tumor DNA (ctDNA) in precision oncology of ovarian cancer
}

\author{
Jaana Oikkonen ${ }^{1} \&$ Sampsa Hautaniemi ${ }^{1}$ \\ ${ }^{1}$ Research Program in Systems Oncology, Faculty of Medicine, University of Helsinki
}

Circulating tumor DNA (ctDNA) is short, typically $<167$ base pairs long DNA fragments ${ }^{1}$ in body fluids, for example, serum and plasma fractions of bloodstream. While mechanisms for ctDNA release are currently poorly understood, a number of articles report good congruence between the mutations and copy-number alterations (75\%-100\%) $)^{2-4}$ identified from ctDNA and representative tissue biopsy. As ctDNA sampling is minimally invasive, ctDNA reflects better cancer heterogeneity in a patient than a tumor biopsy ${ }^{5}$, and in solid tumor cancers often the only option to query genomic landscape of relapsed disease and metastases, it has been welcomed as a basis for precision oncology approaches.

Utility of ctDNA has been well documented in early detection of cancer ${ }^{4,6}$, prognosis ${ }^{7-9}$, and treatment monitoring ${ }^{10,11}$. In CancerSEEK study, $43 \%$ of stage I cancers were detected from ctDNA ${ }^{4}$ and in general, 70\% of cancers were detectable from ctDNA. CtDNA has also been used to detect possible residual disease after primary treatment to identify patients with poor prognosis ${ }^{8}$. Overall, treatment response monitoring through ctDNA allele frequencies is widely adaptable ${ }^{11}$. However, evidence for the use of ctDNA in guiding treatment decisions currently is scarcer ${ }^{12}$. In this editorial we focus on the use of ctDNA in precision oncology in guiding treatments of patients with solid tumor cancers.

ctDNA is used in guiding treatments for non-small cell lung cancer, where EGFR tyrosine kinase inhibitors (TIKs) are given based on EGFR mutations ${ }^{13}$. Currently, the lung cancer ctDNA test is the only FDA approved test for ctDNA-guided treatment. CtDNA is also used for colorectal cancer to target anti-EGFR treatments to specific patients through EGFR and KRAS mutations ${ }^{12}$. In other cancers, ctDNA has been used to guide treatment based on mutations in multiple different genes for example in gastric cancer ${ }^{14,15}$, colorectal cancer $^{16}$ and pancancer settings ${ }^{17-19}$ with positive impact on survival.

We recently published a paper on the use of ctDNA in management of high-grade serous ovarian cancer (HGSOC) ${ }^{2}$. HGSOC is the most frequent and lethal subtype of epithelial ovarian cancer with five-year survival of only $43 \%$. Most patients respond to primary therapy, consisting platinum-based chemotherapy and debulking surgery, but eventually relapse with limited treatment options. All HGSOC patients have TP53 driver mutation, and tumors are characterized by large molecular heterogeneity and abundant copy-number changes. We used targeted sequencing panel of over 600 cancer-related genes and longitudinal sampling to genetically profile 12 patients. In seven patients, actionable targetable mutations and copy-number alterations were identified. For one of them, treatment was changed to include trastuzumab based on the revealed ERBB2 amplification, which resulted in a rapid and dramatic clinical response.

In ovarian cancer, treatment response and detection of relapse are commonly tracked through CA125 through blood. Even with available detection method, ctDNA has shown to detect treatment response more sensitively and relapse earlier than $C A-125^{20,21}$. The tumor burden analyses are based on truncal TP53 mutation allelic frequencies in plasma. The early detection of relapse in 
combination with timely molecular profiling would allow to treat ovarian cancer patients more efficiently.

The main reasons for a relative small number of cases where ctDNA has been used in guiding treatment decisions are as follows. Firstly, the quality of bioinformatics pipelines for ctDNA data analysis has been variable and often poorly documented. Secondly, low amount of ctDNA makes it difficult to obtain enough information and differentiate true signal from noise ${ }^{22}$. Thirdly, timeframe to provide information from ctDNA has been too long to provide timely information to treat patients at the clinic. Lastly, focus has been only on single mutations. It has worked in some cancers, like EGFR mutant lung cancer, but suffers from the heterogeneity between and within patients or possible reversion events.

All the above issues are solvable. Bioinformatics community has responded to the need for open, tailored ctDNA pipelines and several pipelines have been published ${ }^{1,2}$. These new public pipelines for variant calling and filtering enable development of state-of-the-art methods and enable reliable comparisons between studies which may enlighten some of the differences detected earlier ${ }^{23}$. Better protocols for data analysis ensure maximum sensitivity and specificity from the provided sequencing data. Simultaneous development of more sensitive sequencing techniques ${ }^{8}$ enable the detection of lower amounts of ctDNA. Especially the sequencing of early stage cancers and postsurgical minimal residual disease depend on detection of extremely low amounts of ctDNA. New detection techniques have significantly improved the detection limits and there are protocols suitable for detection of low amount of ctDNA in residual disease, as low as 0.0003 allele fractions ${ }^{8}$. The detection limits for larger panels and other than patient-specific mutations are still higher but ongoing efforts are made to overcome the issue also in these assays. Maturation of the protocols has also improved the timeline from sample extraction to passing of clinically relevant information back into the clinics.

Many ctDNA assays provide information on individual mutations, copy-number alterations, or DNA methylations to guide treatment. In studies, where ctDNA-guided matched treatment has been used, not all patients are identified with targetable mutations. A partial explanation is the low number of covered genes and/or low sensitivity. Larger panels exist to detect more rigorous molecular profile and further information on other genetic variation altering the mutation-related response are already being used ${ }^{18}$. When knowledge on molecular variants affecting drug response continue to accumulate, it will be possible to identify more significant, clinically relevant alterations in larger numbers of cancer patients.

\section{REFERENCES}

1. Mouliere F, Chandrananda D, Piskorz AM, et al: Enhanced detection of circulating tumor DNA by fragment size analysis. Sci Transl Med 10:eaat4921, 2018

2. Oikkonen J, Zhang K, Salminen L, et al: Prospective Longitudinal ctDNA Workflow Reveals Clinically Actionable Alterations in Ovarian Cancer [Internet]. JCO Precis Oncol 1-12, 2019Available from: https://ascopubs.org/doi/10.1200/PO.18.00343

3. Yang N, Li Y, Liu Z, et al: The characteristics of ctDNA reveal the high complexity in matching the corresponding tumor tissues. BMC Cancer 18:319, 2018

4. Cohen JD, Li L, Wang Y, et al: Detection and localization of surgically resectable cancers with a multi-analyte blood test. Science 359:926-930, 2018 
5. Abbosh C, Birkbak NJ, Wilson GA, et al: Phylogenetic ctDNA analysis depicts early-stage lung cancer evolution. Nature 545:446-451, 2017

6. Chen $\mathrm{M}$, Zhao $\mathrm{H}$ : Next-generation sequencing in liquid biopsy: cancer screening and early detection. Hum Genomics 13:34, 2019

7. Wang Y, Li L, Cohen JD, et al: Prognostic Potential of Circulating Tumor DNA Measurement in Postoperative Surveillance of Nonmetastatic Colorectal Cancer. JAMA Oncol 5:1118, 2019

8. McDonald BR, Contente-Cuomo T, Sammut S-J, et al: Personalized circulating tumor DNA analysis to detect residual disease after neoadjuvant therapy in breast cancer. Sci Transl Med 11:eaax7392, 2019

9. Reinert T, Henriksen TV, Christensen E, et al: Analysis of Plasma Cell-Free DNA by Ultradeep Sequencing in Patients With Stages I to III Colorectal Cancer. JAMA Oncol 5:1124, 2019

10. Khan KH, Cunningham D, Werner B, et al: Longitudinal Liquid Biopsy and Mathematical Modeling of Clonal Evolution Forecast Time to Treatment Failure in the PROSPECT-C Phase II Colorectal Cancer Clinical Trial. Cancer Discov 8:1-16, 2018

11. Siravegna G, Marsoni S, Siena S, et al: Integrating liquid biopsies into the management of cancer. Nat Rev Clin Oncol 14:531-548, 2017

12. Merker JD, Oxnard GR, Compton C, et al: Circulating tumor DNA analysis in patients with cancer: American society of clinical oncology and college of American pathologists joint review. J Clin Oncol 36:1631-1641, 2018

13. Sacher AG, Paweletz C, Dahlberg SE, et al: Prospective Validation of Rapid Plasma Genotyping for the Detection of EGFR and KRAS Mutations in Advanced Lung Cancer. JAMA Oncol 2:10141022, 2016

14. Kim ST, Banks KC, Lee S-H, et al: Prospective Feasibility Study for Using Cell-Free Circulating Tumor DNA-Guided Therapy in Refractory Metastatic Solid Cancers: An Interim Analysis [Internet]. JCO Precis Oncol 1-15, 2017Available from: http://ascopubs.org/doi/10.1200/PO.16.00059

15. Lee J, Kim ST, Kim K-M, et al: Cell-free DNA sequencing-guided therapy in a prospective clinical trial: NEXT-2 trial-A feasibility analysis. J Clin Oncol 34:11534-11534, 2016

16. Kato S, Schwaederlé MC, Fanta PT, et al: Genomic Assessment of Blood-Derived Circulating Tumor DNA in Patients With Colorectal Cancers: Correlation With Tissue Sequencing, Therapeutic Response, and Survival. JCO Precis Oncol 3:1-16, 2019

17. Rothwell DG, Ayub M, Cook N, et al: Utility of ctDNA to support patient selection for early phase clinical trials: the TARGET study. Nat Med 25:738-743, 2019

18. Sicklick JK, Kato S, Okamura R, et al: Molecular profiling of cancer patients enables personalized combination therapy: the I-PREDICT study. Nat Med 25:744-750, 2019

19. Rodon J, Soria J-C, Berger R, et al: Genomic and transcriptomic profiling expands precision cancer medicine: the WINTHER trial. Nat Med 25:751-758, 2019

20. Parkinson CA, Gale D, Piskorz AM, et al: Exploratory Analysis of TP53 Mutations in Circulating Tumour DNA as Biomarkers of Treatment Response for Patients with Relapsed High-Grade Serous Ovarian Carcinoma: A Retrospective Study. PLoS Med 13:e1002198, 2016

21. Pereira E, Camacho-Vanegas $O$, Anand $S$, et al: Personalized Circulating Tumor DNA Biomarkers Dynamically Predict Treatment Response and Survival In Gynecologic Cancers. PLoS One 10:e0145754, 2015

22. Vowles J V., Zill OA, Eltoukhy H, et al: The Landscape of Actionable Genomic Alterations in CellFree Circulating Tumor DNA from 21,807 Advanced Cancer Patients. Clin Cancer Res 24:35283538, 2018

23. Torga G, Pienta KJ: Patient-Paired Sample Congruence Between 2 Commercial Liquid Biopsy Tests. JAMA Oncol 4:868, 2018 
